\title{
HIGH-IONIZATION MID-INFRARED LINES AS BLACK HOLE MASS AND BOLOMETRIC LUMINOSITY INDICATORS IN ACTIVE GALACTIC NUCLEI
}

\author{
K. M. Dasyra, ${ }^{1}$ L. C. Ho, ${ }^{2}$ L. Armus,${ }^{1}$ P. Ogle, ${ }^{1}$ G. Helou, ${ }^{1}$ B. M. Peterson, ${ }^{3}$ D. Lutz, ${ }^{4}$ H. Netzer,${ }^{5}$ And E. Sturm ${ }^{4}$ \\ Received 2007 October 26; accepted 2007 December 19; published 2008 January 22
}

\begin{abstract}
We present relations of the black hole mass and the optical luminosity with the velocity dispersion and the luminosity of the [ $\mathrm{Ne} \mathrm{V}]$ and the [O IV] high-ionization lines in the mid-infrared (MIR) for 28 reverberationmapped active galactic nuclei. We used high-resolution Spitzer Infrared Spectrograph and Infrared Space Observatory Short Wavelength Spectrometer data to fit the profiles of these MIR emission lines that originate from the narrow-line region of the nucleus. We find that the lines are often resolved and that the velocity dispersion of [ $\mathrm{Ne} \mathrm{v}]$ and [O IV] follows a relation similar to that between the black hole mass and the bulge stellar velocity dispersion found for local galaxies. The luminosity of the [Ne $\mathrm{V}]$ and the [O IV] lines in these sources is correlated with that of the optical $5100 \AA$ continuum and with the black hole mass. Our results provide a means to derive black hole properties in various types of active galactic nuclei, including highly obscured systems.
\end{abstract}

Subject headings: galaxies: active — galaxies: kinematics and dynamics — galaxies: nuclei — galaxies: Seyfert — infrared: galaxies — quasars: emission lines

On-line material: color figures

\section{INTRODUCTION}

Following the relation between the black hole $(\mathrm{BH})$ mass, $M_{\mathrm{BH}}$, and the bulge stellar velocity dispersion, $\sigma_{*}$ (Ferrarese \& Merritt 2000; Gebhardt et al. 2000; Tremaine et al. 2002), a similar relation was found for the velocity dispersion $\sigma$ of the [O III] $5007 \AA$ line (Nelson 2000; Greene \& Ho 2005) that originates from the narrow-line region (NLR) gas of the active galactic nucleus (AGN). The NLR gas is thought to be mostly gravitationally bound to the bulge in high-luminosity AGNs (Nelson \& Whittle 1996; Greene \& Ho 2005; Laor 2007), unlike the broad-line region gas that is virialized due its proximity to the BH (Peterson \& Wandel 1999). This relation is useful for systems in which the stellar absorption lines cannot be observed, e.g., because of dilution of the stellar light by the AGN continuum. In addition to this relation, $M_{\mathrm{BH}}$ and the luminosity $L$ of the $5100 \AA$ optical continuum, $L_{\text {opt }}$, are correlated in AGNs with BH measurements (Kaspi et al. 2000).

There are two main advantages to expanding such relations in the mid-infrared (MIR). The first is that the $\mathrm{Ne} \mathrm{V}$ and $\mathrm{O}$ IV ions emitting at 14.32 and $25.89 \mu \mathrm{m}$ cannot be easily excited by star-forming regions since they have ionization potentials $\chi$ of 97.12 and $54.93 \mathrm{eV}$. The second reason is the low obscuration, which allows for the results to be applied to type 2 AGNs. In this Letter, we investigate for relations between $M_{\mathrm{BH}}$ and $L_{\text {opt }}$ with MIR NLR line velocity dispersions and luminosities.

\section{THE SAMPLE AND THE DATA REDUCTION}

Our sources were selected from a sample of 35 local AGNs that have $M_{\mathrm{BH}}$ measurements from reverberation experiments

\footnotetext{
${ }^{1}$ Spitzer Science Center, California Institute of Technology, Mail Code 2206, 1200 East California Boulevard, Pasadena, CA 91125.

${ }^{2}$ The Observatories of the Carnegie Institution of Washington, 813 Santa Barbara Street, Pasadena, CA 91101.

${ }^{3}$ Department of Astronomy, The Ohio State University, 140 West 18th Avenue, Columbus, $\mathrm{OH} 43210$.

${ }^{4}$ Max-Planck-Institut für extraterrestrische Physik, Postfach 1312, 85741 Garching, Germany.

${ }^{5}$ School of Physics and Astronomy, Raymond and Beverly Sackler Faculty of Exact Sciences, Tel-Aviv University, Tel-Aviv 69978, Israel.
}

(Peterson et al. 2004). Archival high-resolution Spitzer Infrared Spectrograph (IRS) data are available for 28 reverberationmapped AGNs. Of these, eight were previously observed with the Infrared Space Observatory Short Wavelength Spectrometer (SWS) in high resolution (Sturm et al. 2002).

To reduce the IRS data we first averaged all images at the same nod position, rejecting pixels that deviated from the median image by more than 5 times its standard deviation. We computed the uncertainty image and then created the bad-pixel mask and extracted the spectrum of each nod position using the Spitzer data reduction packages IRSCLEAN and SPICE. For the spectral extraction, we assumed a point-source spatial distribution for the high-ionization lines, corresponding to an unresolved NLR. When present, we removed the weak [Cl II] and [Fe II] lines at 14.37 and $25.99 \mu \mathrm{m}, 1000$ and $1200 \mathrm{~km}$ $\mathrm{s}^{-1}$ longward of [ $\left.\mathrm{Ne} \mathrm{v}\right]$ and [O IV]. We fitted the [Ne v] and [O IV] lines and their underlying continua using Gaussian and second-order polynomial functions, respectively, which was a good approximation for most cases. We considered resolved all lines with $\sigma_{m}-\epsilon_{m}>\sigma_{i}+\epsilon_{i}$, where $\sigma_{i}$ is the instrumental resolution $R$ at a given wavelength divided by $2.35, \epsilon_{i}$ is the error of $\sigma_{i}$, and $\sigma_{m}$ is the measured velocity dispersion. The error of $\sigma_{m}, \epsilon_{m}$, is computed as $\left(\epsilon_{\text {std }}^{2}+\epsilon_{i}^{2}\right)^{0.5}$. The quantity $\epsilon_{\text {std }}$ is the standard deviation of the $\sigma_{m}$ values that are obtained when using different polynomial coefficients to describe the local continuum. It encapsulates uncertainties in the continuum level and slope. The instrumental resolution and its error were measured as a function of wavelength using standard IRS calibration targets (P Cygni, HD 190429, HD 174638). The average value of $R$ in the $14.0-18.0 \mu \mathrm{m}$ range is $494 \pm 59 \mathrm{~km}$ $\mathrm{s}^{-1}$. Between 25.0 and $34.2 \mu \mathrm{m}$, it is equal to $503 \pm 63 \mathrm{~km}$ $\mathrm{s}^{-1}$. The intrinsic velocity dispersions of the resolved lines were computed as $\left(\sigma_{m}^{2}-\sigma_{i}^{2}\right)^{0.5}$ and are presented in Table 1. Examples of lines resolved with IRS are given in Figure 1. To complement the Spitzer data and to compute the widths of unresolved lines, we used SWS spectra presented in Sturm et al. (2002). SWS has a higher resolution $\left(R \sim 160 \mathrm{~km} \mathrm{~s}^{-1}\right.$ at $14.32 \mu \mathrm{m}$ and $R \sim 230 \mathrm{~km} \mathrm{~s}^{-1}$ at $\left.25.89 \mu \mathrm{m}\right)$ but lower sensitivity than IRS. It detected only the brightest $[\mathrm{Ne} \mathrm{V}]$ and [O IV] lines, the velocity dispersions of which are presented in 
TABLE 1

Black Hole and MiR Line Properties for Reverberation-Mapped AGNs with High-Resolution Mir Spectra

\begin{tabular}{|c|c|c|c|c|c|c|c|c|c|c|c|c|}
\hline Galaxy & $z$ & & $\left.I_{\mathrm{BH}} M_{\odot}\right)$ & $\left(10^{44}\right.$ & $\begin{array}{l}L_{\mathrm{opt}} \\
\text { ergs }\end{array}$ & $\left.\mathrm{s}^{-1}\right)$ & $\begin{array}{c}\sigma_{*} \\
\left(\mathrm{~km} \mathrm{~s}^{-1}\right)\end{array}$ & $\begin{array}{c}\sigma_{[\mathrm{O} \mathrm{m}]} \\
\left(\mathrm{km} \mathrm{s}^{-1}\right)\end{array}$ & $\begin{array}{c}F_{[\mathrm{Ne} v \mathrm{v}]} \\
\left(10^{-21} \mathrm{\textrm {cm }}^{-2}\right)\end{array}$ & $\begin{array}{c}\sigma_{[\mathrm{Ne} \mathrm{v]}} \\
\left(\mathrm{km} \mathrm{s}^{-1}\right)\end{array}$ & $\begin{array}{c}F_{\left[\mathrm{O}_{\mathrm{IV}]}\right]} \\
\left.\mathrm{W}^{-2} \mathrm{~cm}^{-2}\right)\end{array}$ & $\begin{array}{c}\sigma_{[\mathrm{O} \text { Iv] }} \\
\left(\mathrm{km} \mathrm{s}^{-1}\right)\end{array}$ \\
\hline 3C 120 . & 0.03301 & 5.55 & 2.70 & 1.479 & \pm & 0.272 & $162 \pm 24$ & & $18.06 \pm 0.23$ & $111 \pm 16^{\mathrm{a}}$ & $120.2 \pm 0.8$ & $114 \pm 9^{a}$ \\
\hline Fairall 9 & 0.04702 & 25.5 & 5.6 & 1.778 & \pm & 0.205 & & 181 & $2.60 \pm 0.33$ & $208 \pm 36$ & $6.01 \pm 0.28$ & $215 \pm 39$ \\
\hline IC $4329 A$ & 0.01605 & 0.99 & 1.49 & 0.208 & \pm & 0.024 & $122 \pm 13$ & 234 & $23.63 \pm 1.22$ & $304 \pm 44$ & $100.6 \pm 1.1$ & $278 \pm 30$ \\
\hline Mrk 279 . & 0.03045 & 3.49 & 0.92 & 0.756 & \pm & 0.087 & $197 \pm 12$ & 247 & $3.28 \pm 0.23$ & $247 \pm 35$ & $10.82 \pm 0.29$ & \\
\hline Mrk 509 & 0.0344 & 14.3 & 1.2 & 1.905 & \pm & 0.351 & & 221 & $6.29 \pm 0.26$ & $\ldots$ & $28.73 \pm 0.29$ & $133 \pm 34^{\mathrm{a}}$ \\
\hline Mrk 590 & 0.02638 & 4.75 & 0.74 & 0.646 & \pm & 0.134 & $192 \pm 10$ & 170 & $1.01 \pm 0.16$ & & $3.39 \pm 0.17$ & $197 \pm 30$ \\
\hline NGC 3227 & 0.00386 & 4.22 & 2.14 & 0.024 & \pm & 0.002 & $134 \pm 6$ & 206 & $23.13 \pm 0.35$ & $212 \pm 34$ & $65.37 \pm 0.59$ & $210 \pm 30$ \\
\hline NGC 3783 . & 0.00973 & 2.98 & 0.54 & 0.182 & \pm & 0.017 & $95 \pm 10$ & 98 & $15.24 \pm 1.00$ & $133 \pm 21^{\mathrm{a}}$ & $39.78 \pm 0.84$ & $142 \pm 25^{\mathrm{a}}$ \\
\hline NGC 4051 . & 0.00234 & 0.191 & 0.078 & 0.0085 & \pm & 0.0010 & $87 \pm 5$ & 81 & $11.13 \pm 0.25$ & $\ldots$ & $33.69 \pm 0.52$ & $235 \pm 32$ \\
\hline NGC 4151 & 0.00332 & 4.57 & 0.52 & 0.076 & \pm & 0.040 & $95 \pm 7$ & 181 & $77.64 \pm 1.55$ & $139 \pm 26^{\mathrm{a}}$ & $243.5 \pm 1.5$ & $169 \pm 13^{\mathrm{a}}$ \\
\hline NGC 4593 & 0.00900 & 0.98 & 0.21 & 0.123 & \pm & 0.040 & $129 \pm 15$ & 109 & $3.48 \pm 0.35$ & & $13.79 \pm 0.86$ & $\ldots$ \\
\hline NGC 5548 & 0.01717 & 6.54 & 0.26 & 0.324 & \pm & 0.324 & $192 \pm 15$ & 174 & $3.23 \pm 0.21$ & $200 \pm 33$ & $12.80 \pm 0.66$ & \\
\hline NGC 7469 . & 0.01632 & 1.22 & 0.14 & 0.525 & \pm & 0.024 & $142 \pm 3$ & 153 & $12.07 \pm 0.63$ & & $40.63 \pm 3.06$ & $179 \pm 22^{\mathrm{a}}$ \\
\hline PG $0003+199$ & 0.02578 & 1.42 & 0.37 & 0.724 & \pm & 0.067 & $\ldots$ & 119 & $1.21 \pm 0.30$ & $253 \pm 50$ & $6.44 \pm 0.28$ & \\
\hline PG $0026+129$. & 0.142 & 39.3 & 9.6 & 10.47 & \pm & 1.45 & $\ldots$ & $\ldots$ & $0.38 \pm 0.12$ & $\ldots$ & $2.71 \pm 0.38$ & $291 \pm 51$ \\
\hline PG $0804+761 \ldots \ldots$ & 0.1 & 69.3 & 8.3 & 8.710 & \pm & 1.604 & $\ldots$ & $\ldots$ & $<0.40$ & & $1.93 \pm 0.25$ & $308 \pm 57$ \\
\hline PG $0844+349 \ldots \ldots$ & 0.064 & 9.24 & 3.81 & 2.239 & \pm & 0.206 & $\ldots$ & $\ldots$ & $<0.72$ & $267 \pm 56$ & $1.47 \pm 0.21$ & $\ldots$ \\
\hline PG $0953+414 \ldots \ldots$ & 0.2341 & 27.6 & 5.9 & 16.60 & \pm & 2.29 & $\ldots$ & 231 & $<0.48$ & & $<1.14$ & \\
\hline PG $1211+143 \ldots \ldots$ & 0.0809 & 14.6 & \pm 4.4 & 5.623 & \pm & 0.777 & $\ldots$ & $\ldots$ & $0.53 \pm 0.14$ & $252 \pm 58$ & $1.45 \pm 0.26$ & $209 \pm 40$ \\
\hline PG $1226+023$. & 0.1583 & 88.6 & \pm 18.7 & 91.20 & \pm & 10.50 & $\ldots$ & 321 & $2.61 \pm 0.42$ & $504 \pm 64$ & $9.17 \pm 0.33$ & $423 \pm 61$ \\
\hline PG $1229+204$. & 0.0603 & 7.32 & $\pm \quad 3.52$ & 1.202 & \pm & 0.138 & $162 \pm 32$ & $\ldots$ & $0.93 \pm 0.07$ & 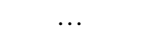 & $2.59 \pm 0.45$ & $\ldots$ \\
\hline PG $1307+085$. & 0.155 & 44.0 & \pm 12.3 & 7.586 & \pm & 0.700 & $\ldots$ & 219 & $0.67 \pm 0.06$ & $262 \pm 38$ & $1.96 \pm 0.33$ & \\
\hline PG $1411+442$. & 0.0896 & 44.3 & \pm 14.6 & 4.266 & \pm & 0.393 & $\cdots$ & 175 & $0.64 \pm 0.09$ & $284 \pm 54$ & $1.87 \pm 0.24$ & $228 \pm 52$ \\
\hline PG $1426+015 \ldots \ldots$ & 0.0865 & 129.8 & \pm 38.5 & 5.248 & \pm & 0.846 & $185 \pm 67$ & 197 & $1.20 \pm 0.17$ & $292 \pm 46$ & $4.37 \pm 0.56$ & $250 \pm 38$ \\
\hline PG $1613+658 \ldots \ldots$ & 0.129 & 27.9 & \pm 12.9 & 9.550 & \pm & 1.099 & & 230 & $1.16 \pm 0.21$ & $202 \pm 43$ & $5.28 \pm 0.25$ & $206 \pm 34$ \\
\hline PG $1617+175 \ldots \ldots$ & 0.11244 & 59.4 & \pm 13.8 & 3.020 & \pm & 0.556 & $183 \pm 47$ & $\ldots$ & $<0.77$ & $\ldots$ & $<1.62$ & $\ldots$ \\
\hline PG $1700+518 \ldots \ldots$ & 0.292 & 78.1 & \pm 17.4 & 42.66 & \pm & 2.95 & & 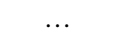 & $<0.39$ & & $<1.82$ & \\
\hline PG $2130+099$. & 0.063 & 45.7 & \pm 5.5 & 2.884 & \pm & 0.266 & $172 \pm 46$ & $\ldots$ & $4.06 \pm 0.20$ & $318 \pm 44$ & $10.00 \pm 0.31$ & $\ldots$ \\
\hline
\end{tabular}

Note. - The $M_{\mathrm{BH}}$ and $L_{\mathrm{opt}}$ values are from Peterson et al. (2004), Bentz et al. (2006, 2007), and Denney et al. (2006). The $\sigma_{*}$ values were extracted from the $\mathrm{Ca}$ II triplet (Onken et al. 2004; Nelson et al. 2004) in the Seyfert galaxies and from the CO band heads (Dasyra et al. 2007) in the QSOs. The $\sigma_{[\mathrm{OO} m]}$ values are from Peterson et al. (2004), Shields et al. (2003, 2006), and Bonning et al. (2005). For sources with two measurements of $\sigma$, the average value was used. The MIR line detection limit was set to 3 times the noise standard deviation.

${ }^{a}$ The velocity dispersions of these lines were extracted from SWS spectra presented in Sturm et al. (2002).

Table 1. To fit the relations between the MIR line and the $\mathrm{BH}$ properties, we used the FITEXY algorithm that takes into account the error bars in both axes (Press et al. 1992) and that treats all variables symmetrically (Tremaine et al. 2002).

\section{RESULTS}

\subsection{Correlations between $M_{\mathrm{BH}}$ and the Velocity Dispersion of $[\mathrm{Ne} \mathrm{v}]$ and $[\mathrm{O} \mathrm{IV}]$}

The $M_{\mathrm{BH}^{-}} \sigma$ relation of the MIR high-ionization lines is presented in Figure 2. Its best-fit solution is

$$
\log \left(\frac{M_{\mathrm{BH}}}{M_{\odot}}\right)=\alpha_{1}+\beta_{1} \times \log \left(\frac{\sigma}{200 \mathrm{~km} \mathrm{~s}^{-1}}\right),
$$

with parameter values corresponding to $\alpha_{1}=7.86 \pm 0.09$ and $\beta_{1}=4.31 \pm 0.63$ for $[\mathrm{Ne} \mathrm{v}]$ and $\alpha_{1}=8.04 \pm 0.08$ and $\beta_{1}=4.89 \pm 0.63$ for [O IV]. The scatter values of the relation along the $M_{\mathrm{BH}}$ axis are 0.55 and $0.51 \mathrm{dex}$ for $[\mathrm{Ne} \mathrm{v}$ ] and [O IV], respectively. Its Spearman rank coefficients are 0.66 and 0.77 , assigning probabilities $p$ of $5.0 \times 10^{-3}$ and $8.3 \times$ $10^{-4}$ to the null hypothesis that the variables are correlated by chance. We excluded all sources with $M_{\mathrm{BH}}<10^{7} M_{\odot}$ from this fit since the IRS resolution is insufficient to provide $\sigma$ measurements on the left-hand side of the relation and since no SWS measurements are available for such $M_{\mathrm{BH}}$ values. Moreover, IC 4329A is not used in the fit since its light curves are not indicative of the presence of a BH (Peterson et al. 2004). Given that the best-fit slope agrees within the errors with that of the Tremaine et al. (2002) formula, the latter can be alter- natively used to derive $M_{\mathrm{BH}}$ from $\sigma_{[\mathrm{Ne} \mathrm{v}]}$ and $\sigma_{[\mathrm{O} \mathrm{Iv}]}$. If we fix the slope to $\beta_{1}=4.02$ and solve for $\alpha_{1}$ using the IDL routine MPFIT, we find that $\alpha_{1}=7.90 \pm 0.25$ and $\alpha_{1}=8.11 \pm$ 0.26 for $[\mathrm{Ne} \mathrm{v}]$ and [O IV].

\subsection{Correlations between $L_{\mathrm{opt}}, M_{\mathrm{BH}}$, and the Luminosity of [Ne v] and [O IV]}

Following the suggestion that the luminosity of [O III] can be used as a proxy of $L_{\text {opt }}$ (Heckman et al. 2004), we find that

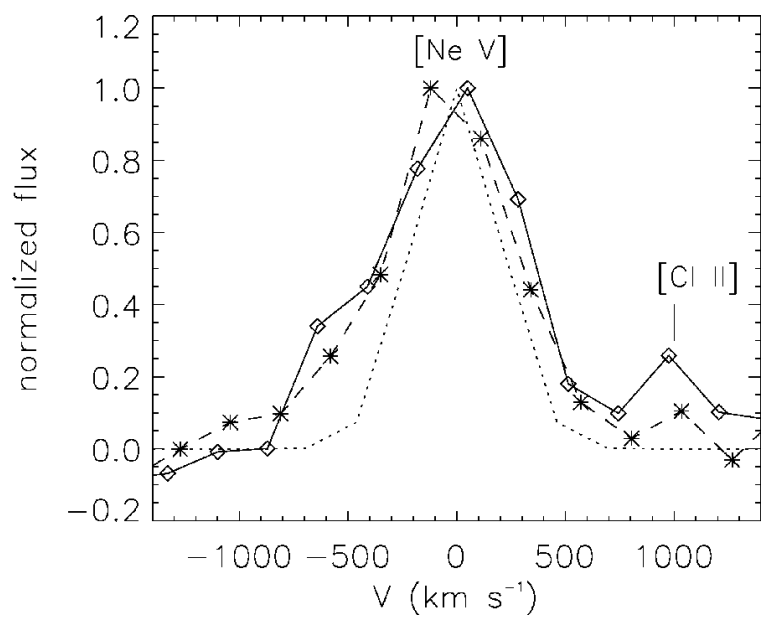

FIG. 1.-Examples of lines resolved with IRS. The [Ne v] profile is given for PG 2130+099 (solid line, diamonds) and Mrk 279 (dashed line, stars). The resolution element is plotted as a dotted line. 

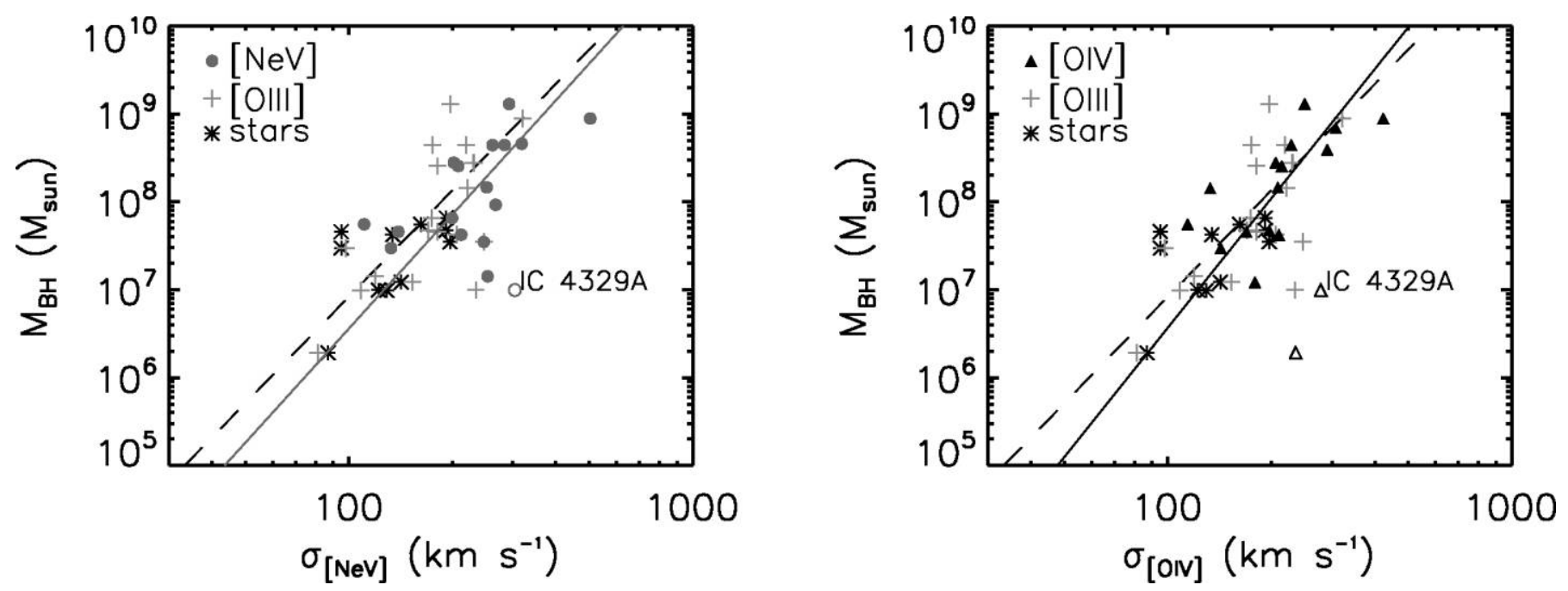

FIG. 2.-Relation between $M_{\mathrm{BH}}$ and the velocity dispersion of the MIR NLR lines [Ne v] (left, circles) and [O IV] (right, triangles). The solid line corresponds to the best-fit solution, and the open symbols correspond to sources that have been excluded from the fit. For comparison, we overplot the Tremaine et al. (2002) relation (dashed line) and the stellar, Ca II, velocity dispersion measurements of the reverberation-mapped AGNs (stars; Onken et al. 2004; Nelson et al. 2004). The crosses correspond to $\sigma_{[\mathrm{O} \mathrm{m}]}$. [See the electronic edition of the Journal for a color version of this figure.]

$L_{[\mathrm{Nev}]}$ and $L_{[\mathrm{O} \text { vv }]}$ are related to the $5100 \AA$ continuum luminosity (Fig. 3) as

$$
\log \left(\frac{L_{\mathrm{opt}}}{10^{44} \operatorname{ergs~s}^{-1}}\right)=\alpha_{2}+\beta_{2} \times \log \left(\frac{L_{\mathrm{MIR} \mathrm{line}}}{10^{41} \operatorname{ergs~s}^{-1}}\right)
$$

and they are computed for $H_{0}=70 \mathrm{~km} \mathrm{~s}^{-1} \mathrm{Mpc}^{-1}, \Omega_{m}=$ 0.3 , and $\Omega_{\text {total }}=1$. The best-fit parameter values are $\alpha_{2}=$ $-0.04 \pm 0.01$ and $\beta_{2}=1.17 \pm 0.02$ for $[\mathrm{Ne} \mathrm{v}]$ and $\alpha_{2}=$ $-0.55 \pm 0.01$ and $\beta_{2}=1.15 \pm 0.01$ for [O IV]. The scatter values of the relation are 0.46 and 0.47 dex and its Spearman rank coefficients are 0.85 and 0.81 for [Ne v] and [O IV], respectively, corresponding to probabilities $p$ of $3.7 \times 10^{-7}$ and $7.5 \times 10^{-7}$. The relations continue to hold when plotting the $5100 \AA$ continuum versus the MIR line fluxes (Fig. 3). In the flux-flux diagram, the only outlier is PG $1226+023$ (3C 273), which is the brightest Palomar-Green QSO (Schmidt \& Green 1983).

A correlation similar to that found in the optical between

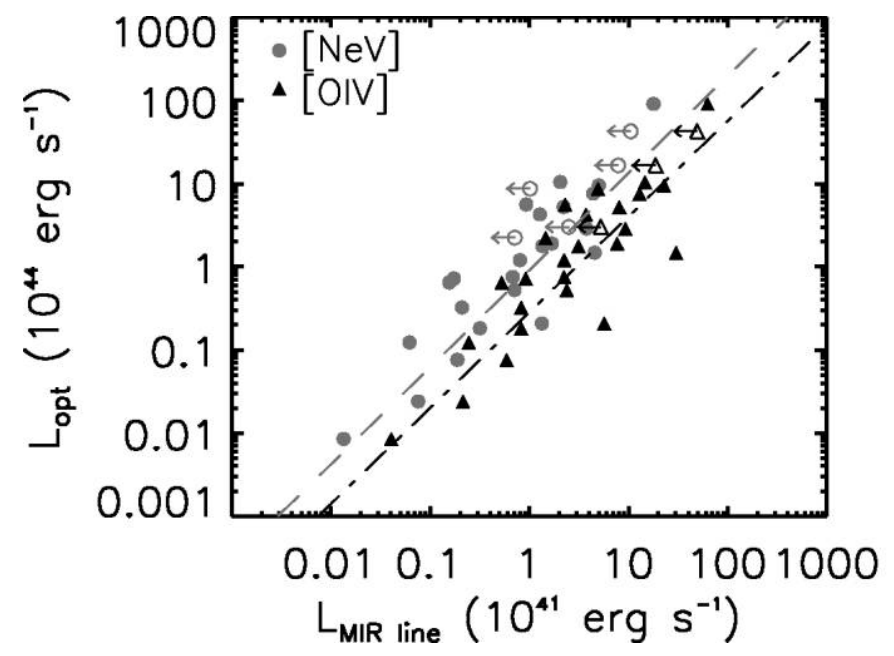

$M_{\mathrm{BH}}$ and $L_{\mathrm{opt}}$ exists in the MIR between $M_{\mathrm{BH}}$ and $L_{[\mathrm{Ne} \text { v] }}$ or $L_{[\mathrm{O} \text { iv] }}$ (Fig. 4). Its best fit is

$$
\log \left(\frac{M_{\mathrm{BH}}}{M_{\odot}}\right)=\alpha_{3}+\beta_{3} \times \log \left(\frac{L_{\mathrm{MIR} \text { line }}}{10^{41} \operatorname{ergs~s}^{-1}}\right)
$$

with $\alpha_{3}=8.09 \pm 0.02$ and $\beta_{3}=0.74 \pm 0.03$ for $[\mathrm{Ne} \mathrm{v}$ ] and $\alpha_{3}=7.72 \pm 0.02$ and $\beta_{3}=0.77 \pm 0.03$ for [O IV]. Its scatter values are 0.46 and 0.50 dex and its Spearman rank coefficients are 0.76 and 0.69 with $p=2.5 \times 10^{-5}$ and $p=1.3 \times 10^{-4}$ for [Ne v] and [O IV], respectively. We note that upper limits have not been used for the fit.

\section{DISCUSSION: ORIGIN AND CONDITIONS FOR THE APPLICATION OF THE RELATIONS}

The relations between $\sigma_{[\mathrm{Ne} \mathrm{v}]}, \sigma_{[\mathrm{O} \mathrm{Ivv}}$, and $M_{\mathrm{BH}}$ indicate that the NLR gas kinematics are primarily determined by the potential of the bulge, as it is also believed based on the [O III] line

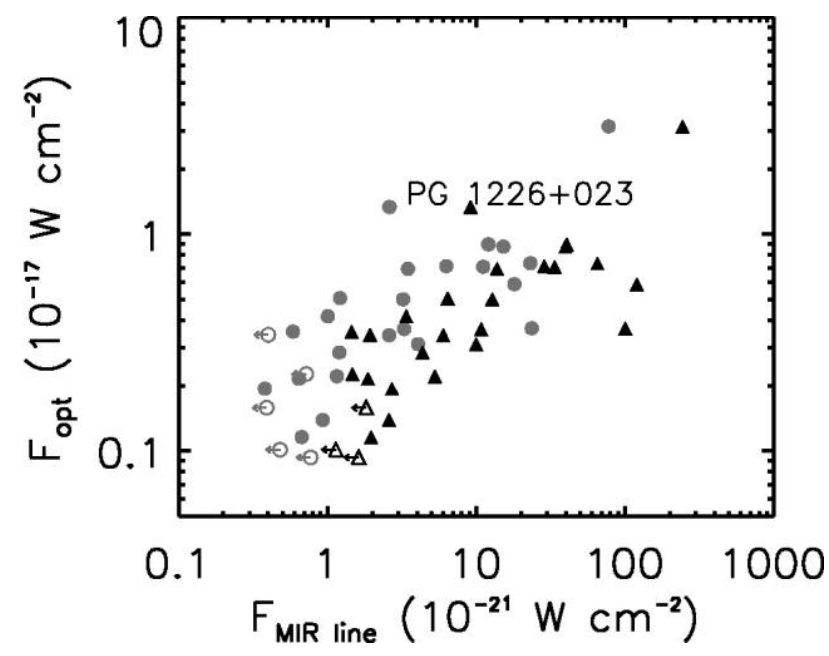

Fig. 3.-Luminosity (left) and flux (right) of the optical continuum scale with those of the MIR NLR lines. The best-fit solution to the [Ne v] data points (circles) is plotted with a dashed line and that to the [O IV] data points (triangles) is plotted with a dash-dotted line. The open symbols correspond to limits, not used for the fit. [See the electronic edition of the Journal for a color version of this figure.] 


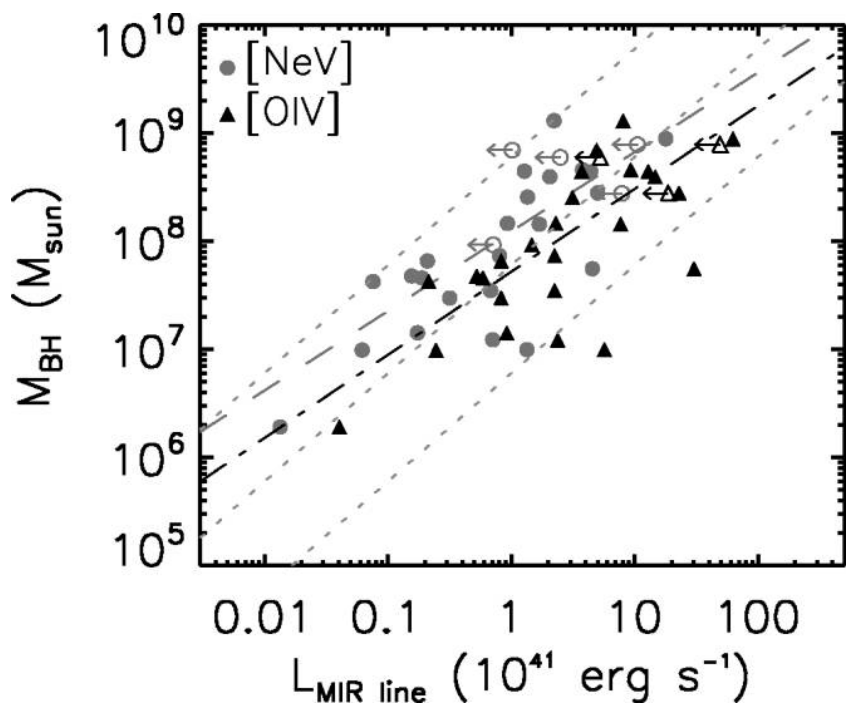

FIG. 4.-Relation between $M_{\mathrm{BH}}$ and the luminosity of the MIR NLR lines. The symbols are as in Fig. 3. The dotted lines correspond to a fixed Eddington rate of $0.01,0.1$, and 1 from top to bottom. They were horizontally shifted by a factor of 1000 to overlap with the luminosity range of the MIR NLR lines. [See the electronic edition of the Journal for a color version of this figure.]

profiles (Whittle 1992; Nelson \& Whittle 1996; Greene \& Ho 2005). However, $\sigma_{[\mathrm{Ne} \mathrm{v}]}$ and $\sigma_{[\mathrm{O} \mathrm{Iv}]}$ are on average 67 and $62 \mathrm{~km}$ $\mathrm{s}^{-1}$ higher than $\sigma_{*}$, and 51 and $32 \mathrm{~km} \mathrm{~s}^{-1}$ higher than $\sigma_{[\mathrm{O} \mathrm{II]}}$. Such discrepancies could be attributed to an increase of the line width with increasing $\chi$, which is often found for optical NLR lines (e.g., Osterbrock 1981; Whittle 1985b). It is possible that ions with high ionization potentials have high velocity dispersions because they are located in NLR clouds that are close to the BH sphere of influence. Since the O IV and, mostly, the $\mathrm{Ne} \mathrm{v}$ ions are predominantly excited by AGNs, the MIR $M_{\mathrm{BH}^{-}} \sigma$ relation can be applied even in systems that undergo starbursts. It can also be applied in type 2 AGNs since the lines suffer little from extinction. Moreover, $\sigma_{[\mathrm{Ne} \mathrm{v}]}$ and $\sigma_{[\mathrm{O} \text { Iv }]}$ can be used as surrogates for $\sigma_{*}$ in environments where measuring $\sigma_{*}$ is hard, such as in bright QSOs. However, the relation does not necessarily hold for AGNs with strong winds and jets such as luminous radio sources (Whittle 1992) and for AGNs with high Eddington rates, $\eta_{\text {Edd }}$ (Greene \& Ho 2005). Such systems can be recognized by the asymmetric wings or the high kurtosis of their line profiles (Whittle 1985a, 1992).

The luminosities of the broad lines (Baldwin 1977) and the narrow lines (Fig. 3) scale with $L_{\text {opt }}$ and with the bolometric AGN luminosity, $L_{\text {bol }}$, since they depend on the absolute accretion rate onto the central object. Specifically, $L_{\mathrm{bol}}$ is equal to $x_{\text {opt }} L_{\text {opt }}, x_{[\mathrm{Ne} \mathrm{v}]} L_{[\mathrm{Ne} \mathrm{v}]}$, and $x_{[\mathrm{O} \mathrm{Iv}]} L_{[\mathrm{O} \mathrm{Iv}]}$, where $x$ is a wavelengthdependent bolometric correction factor. For $x_{\mathrm{opt}}=9$ (Kaspi et al. 2000; Marconi et al. 2004) and for the median values of $L_{\mathrm{op}} / L_{[\mathrm{Ne} \mathrm{v}]}$ and $L_{\mathrm{opp}} / L_{[\mathrm{O} \text { iv }]}$ in our sample, we find that $x_{[\mathrm{Ne} \mathrm{v}]}=$ 13,000 and $x_{[\mathrm{O} \text { iv }]}=4500$. That the relation does not have a slope of unity could indicate that $x_{[\mathrm{Ne} \mathrm{v}]}$ and $x_{[\mathrm{O} \mathrm{Iv}]}$ depend on $L$ in a manner different than $x_{\text {opt }}$ does. Netzer et al. (2006) found that the equivalent width of [O III] decreases with increasing $L_{\text {opt }}$. This behavior could be attributed to a different geometric distribution of NLR clouds around the AGN at different luminosities.

Whether the [ $\mathrm{Ne} \mathrm{v}$ ] or [O IV] luminosity of a source can be used to derive its $M_{\mathrm{BH}}$ partly depends on its Eddington rate. To illustrate how changes of $\eta_{\text {Edd }}$ affect the relation between the luminosity of a MIR line and $M_{\mathrm{BH}}$, we overplot lines of constant $\eta_{\text {Edd }}$ in Figure 4 . The more quiescent a source is, the more its position is shifted to the top left corner of this diagram. Equation (3) is valid within the $\eta_{\text {Edd }}$ range $0.003-0.6$ that our sources span and implies that the mass of a $\mathrm{BH}$ determines its luminosity output. Its advantage is that it can be applied to sources with obscured optical continua or underestimated [O III] luminosities, such as type 2 AGNs (Netzer et al. 2006). However its scatter is likely to be larger than that presented in $\S 3.2$ since the Eddington rates of the reverberation-mapped AGNs are not necessarily representative of those of all local AGNs.

We conclude that the MIR NLR gas kinematics trace $M_{\mathrm{BH}}$ in local reverberation-mapped AGNs in a manner similar to stellar kinematics. The [Ne v] and [O IV] line widths can be used to estimate $M_{\mathrm{BH}}$. The calibration of $L_{[\mathrm{Ne} \mathrm{v}]}$ and $L_{[\mathrm{O} \text { Iv }]}$ to $L_{\mathrm{opt}}$ provides a new method to compute bolometric luminosities, black hole masses, and Eddington rates in various types of AGNs, including highly obscured systems, albeit with a large scatter.

This work was based on observations made with the Spitzer Space Telescope and was supported by NASA through an award issued by JPL/Caltech.

\section{REFERENCES}

Baldwin, J. A. 1977, ApJ, 214, 679

Bentz, M. C., et al. 2006, ApJ, 651, 775 2007, ApJ, 662, 205

Bonning, E. W., Shields, G. A., Salviander, S., \& McLure, R. J. 2005, ApJ, 626, 89

Dasyra, K. M., et al. 2007, ApJ, 657, 102

Denney, K. D., et al. 2006, ApJ, 653, 152

Ferrarese, L., \& Merritt, D. 2000, ApJ, 539, L9

Gebhardt, K., et al. 2000, ApJ, 539, L13

Greene, J. E., \& Ho, L. C. 2005, ApJ, 627, 721

Heckman, T. M., Kauffmann, G., Brinchmann, J., Charlot, S., Tremonti, C., \& White, S. D. M. 2004, ApJ, 613, 109

Kaspi, S., Smith, P. S., Netzer, H., Maoz, D., Jannuzi, B. T., \& Giveon, U. 2000, ApJ, 533, 631

Laor, A. 2007, in ASP Conf. Ser. 373, The Central Engine of Active Galactic Nuclei, ed. L. C. Ho \& J.-M. Wang (San Francisco: ASP), 384

Marconi, A., Risaliti, G., Gilli, R., Hunt, L. K., Maiolino, R., \& Salvati, M. 2004, MNRAS, 351, 169

Nelson, C., Green, R. F., Bower, G., Gebhardt, K., \& Weistrop, D. 2004, ApJ, 615,652

Nelson, C. H. 2000, ApJ, 544, L91
Nelson, C. H., \& Whittle, M. 1996, ApJ, 465, 96

Netzer, H., Mainieri, V., Rosati, P., \& Trakhtenbrot, B. 2006, A\&A, 453, 525

Onken, C. A., Ferrarese, L., Merritt, D., Peterson, B. M., Pogge, R. W., Vestergaard, M., \& Wandel, A. 2004, ApJ, 615, 645

Osterbrock, D. E. 1981, ApJ, 246, 696

Peterson, B. M., \& Wandel, A. 1999, ApJ, 521, L95

Peterson, B. M., et al. 2004, ApJ, 613, 682

Press, W. H., Teukolsky, S. A., Vetterling, W. T., \& Flannery, B. P. 1992, Numerical Recipes (Cambridge: Cambridge Univ. Press)

Schmidt, M., \& Green, R. 1983, ApJ, 269, 352

Shields, G. A., Gebhardt, K., Salviander, S., Wills, B. J., Xie, B., Brotherton, M. S., Yuan, J., \& Dietrich, M. 2003, ApJ, 583, 124

Shields, G. A., Menezes, K. L., Massart, C. A., \& Vanden Bout, P. 2006, ApJ, 641,683

Sturm, E., Lutz, D., Verma, A., Netzer, H., Sternberg, A., Moorwood, A., Oliva, E., \& Genzel, R. 2002, A\&A, 393, 821

Tremaine, S., et al. 2002, ApJ, 574, 740

Whittle, M. 1985a, MNRAS, 213, 33 1985b, MNRAS, 216, 817 1992, ApJ, 387, 109 